\section{Fatores associados ao déficit nutricional em crianças residentes em uma área de prevalência elevada de insegurança alimentar}

\section{Factors associated with malnutrition in children living in food insecurity households}

\section{Gabriela dos Santos Barroso'}

Rosely Sichieri²

\section{Rosana Salles-Costa ${ }^{3}$}

1 Programa de Pós-Graduação em Nutrição - Instituto de Nutrição Josué de Castro - UFRJ; Universidade Federal Fluminense - Hospital Universitário Antonio Pedro.

${ }^{2}$ Instituto de Medicina Social/ Departamento de Epidemiologia - UERJ; Núcleo de Epidemiologia e Biologia da Nutrição (NEBIN).

${ }^{3}$ Instituto de Nutrição Josué de Castro/ Departamento de Nutrição Social e Aplicada - UFRJ.

Artigo baseado em dissertação de Mestrado - Universidade Federal do Rio de Janeiro/Instituto de Nutrição Josué de Castro, defendida em Março de 2006: "Associação entre indicadores socioeconômicos, perfil de saúde e estado nutricional de uma amostra representativa de crianças, na faixa etária de 6 a 30 meses, residentes no segundo distrito do Município de Duque de Caxias/ Rio de Janeiro".

Auxílio Financeiro: Conselho Nacional de Desenvolvimento Científico e Tecnológico -CNPq (processo $n^{\circ}$ 503139/2003-3) e Instituto Nacional de Câncer (Ministério da Saúde).

Agradecimentos: Ao CNPq (processo n 503139/2003-3) e Instituto Nacional de Câncer (Ministério da Saúde) pelo apoio financeiro. Os autores agradecem a colaboração de Luiz Alberto Matzembacher (IBGE) pela elaboração do banco de dados e apoio logístico na etapa de digitação dos questionários.

Aprovado pelo Comitê de Ética em Pesquisa da Universidade do Estado do Rio de Janeiro.

Correspondência: Gabriela dos Santos Barroso. Instituto de Nutrição Josué de Castro - UFRJ Universidade Federal Fluminense - Hospital Universitário Antonio Pedro. Rua Bandeira de Melo, 111 - Realengo, RJ. CEP 21760-010. E-mail : biasbarroso@ig.com.br

Endereço para correspondência: Gabriela dos Santos Barroso - Rua Bandeira de Melo, 111 - Realengo, Rio de Janeiro - RJ. CEP: 21760-010.

\section{Resumo}

Objetivo: Avaliou-se a influência dos indicadores socioeconômicos e do perfil de saúde no estado nutricional de uma amostra representativa de crianças, na faixa etária de seis a trinta meses, do $2^{\circ}$ distrito do município de Duque de Caxias, no Rio de Janeiro. Métodos: Estudo transversal, conduzido por visita domiciliar e com amostragem probabilística de 1.085 domicílios. A amostra foi composta por 402 domicílios com crianças. A avaliação do estado nutricional incluiu peso e comprimento/estatura. Os índices antropométricos peso-para-idade $(\mathrm{P} / \mathrm{I})$, comprimento/estatura-para-idade (E/I) e peso-para-comprimento/estatura (P/E), expressos em escores-z, foram utilizados para a classificação nutricional. Utilizou-se a regressão logística com modelo hierárquico para identificar os fatores socioeconômicos (determinantes básicos e intermediários) e de saúde das crianças (determinantes imediatos) associados ao estado nutricional infantil. Resultados: A prevalência de déficit nutricional foi de 2,8\%, 8,6\% e 3,3\%, considerando-se os respectivos índices $\mathrm{P} / \mathrm{I}$, $\mathrm{E} / \mathrm{I} \mathrm{e} \mathrm{P/E}$. A renda familiar mensal per capita associou-se ao déficit de P/E. O baixo peso ao nascer foi fortemente associado a déficits dos índices E/I e P/E, independentemente da renda e de outros determinantes básicos e intermediários. Conclusão: O baixo peso ao nascer foi o principal fator associado ao déficit nutricional infantil e ações visando à redução desse determinante podem contribuir na reversão do quadro de desnutrição em populações onde persiste a desigualdade social.

Palavras-chave: Indicadores socioeconômicos. Estado nutricional infantil. Baixo peso ao nascer. Insegurança alimentar. 


\section{Abstract}

Objective: The influence of socioeconomic and health indicators on the nutritional status was evaluated in a probabilistic sample of children aged 6 to 30 months, living in Duque de Caxias - Rio de Janeiro. Methods: Cross-sectional study of a probabilistic household sample comprised of 1,085 households. The total size sample was 402 households with children. The analysis of the nutritional status included weight and lengh/stature. Anthropometric indexes weight-for-age (WAZ), length-for-age (HAZ) and weight-for-length (WHZ) expressed in $\mathrm{z}$-scores were used to evaluate nutritional status. Logistic regression and a hierarchical approach were used to identify socioeconomic factors (basic and intermediate determinants) and health of children (immediate determinants) associated with nutritional status of infants. Results: The prevalence of malnutrition was $2.8 \%, 8.6 \%$ and $3.3 \%$ for theWAZ, HAZ and WHZ indexes, respectively. Per capita family income was significantly associated with WHZ. Low birth weight was strongly associated with low HAZ and WHZ indexes, regardless of income and other basic and intermediate determinants. Conclusion: Low birth weight was the most important factor associated with nutritional deficit in children. Actions aiming to reduce low birth weight can contribute to reverse malnutrition in populations in which social inequality persists.

Keywords: Socioeconomic indicators. Nutritional status of children. Low birth weight and food insecurity.

\section{Introdução}

Os indicadores socioeconômicos representam um dos principais fatores associados com o estado nutricional de crianças e são considerados determinantes básicos e imediatos da desnutrição infantil pelo Fundo das Nações Unidas para a Infância ${ }^{1}$. Entre esses indicadores, a evolução do poder aquisitivo das famílias e a progressão da escolaridade dos membros destas repercutem diretamente na tendência secular das condições de saúde na infância ${ }^{2}$. Do poder aquisitivo dependem, por exemplo, a disponibilidade de alimentos, a qualidade do ambiente e o acesso a serviços essenciais como os de saneamento e os de assistência à saúde. Da escolaridade dos familiares, sobretudo da escolaridade materna, depende a utilização mais ou menos eficiente (do ponto de vista do cuidado infantil) da renda e dos serviços públicos ${ }^{3}$.

Além destes determinantes definidos pelo UNICEF cabe acrescentar a importância dos indicadores relacionados à saúde da criança, que também influenciam o estado nutricional infantil, destacando-se o peso ao nascer e as doenças infecciosas comuns da infância (diarréia e infecção).

A nutrição adequada é um dos fatores de maior impacto na saúde infantil, principalmente pela influência decisiva que o estado nutricional exerce sobre os riscos de morbimortalidade e sobre o crescimento e o desenvolvimento ${ }^{4}$. Segundo relatório publicado pela Organização Mundial da Saúde (OMS), em 2000, nos países em desenvolvimento, cerca de $35 \%$ das crianças abaixo dos cinco anos de idade apresentam déficit de estatura, sendo que, na América do Sul, a prevalência deste déficit era de $9,3 \%{ }^{5}$.

Neste campo de investigação surge o conceito de Segurança Alimentar e Nutricional definido como a garantia de acesso contínuo à quantidade e qualidade suficientes de alimentos, obtido por meio socialmente aceitável, de forma a assegurar o bem-estar e a saúde dos indivíduos ${ }^{6}$. Sua utilização nos estudos populacionais vem ocupando de forma crescente a agenda pública do 
Brasil, devido ao seu papel como marcador de desigualdades inerentes ao sistema econômico. Recentemente, Pimentel ${ }^{7}$ (2007) avaliou a insegurança alimentar na mesma população deste estudo e encontrou que as famílias com crianças de seis a trinta meses de idade apresentaram $72 \%$ de insegurança alimentar, sendo $12 \%$ consideradas com insegurança alimentar grave, segundo a classificação da Escala Brasileira de Insegurança Alimentar ${ }^{8}$.

Esse resultado indicou a importância de se avaliar a influência dos indicadores socioeconômicos e do perfil de saúde no estado nutricional de uma amostra representativa de crianças, na faixa etária de seis a trinta meses, do $2^{\circ}$ distrito do município de Duque de Caxias, no Rio de Janeiro.

\section{Métodos}

Trata-se de um estudo transversal de base domiciliar inserido no projeto "Avaliação do estado nutricional, hábitos alimentares e insegurança alimentar no município de Duque de Caxias, Rio de Janeiro: desenvolvimento de um instrumento simplificado para avaliação de consumo alimentar saudável", realizado em parceria com o Instituto de Medicina Social da UERJ, Instituto de Nutrição Josué de Castro da UFRJ, Faculdade de Nutrição da UFF, Instituto Nacional de Câncer (MS) e IBGE/ENCE.

O Município de Duque de Caxias localiza-se na Região Metropolitana do Rio de Janeiro, a 27 km de distância da Capital do Estado e apresenta uma área de $468,3 \mathrm{~km}^{2}$. O segundo distrito de Duque de Caxias (Campos Elíseos) foi selecionado para o presente estudo por ser um dos distritos mais pobres do município, apresentando uma população residente de 243.767 habitantes, sendo $6,4 \%$ na faixa etária de 0 a 2 anos.

Utilizou-se amostragem probabilística estimada em 1125 domicílios particulares permanentes (DPP) do segundo distrito do município de Duque de Caxias, conglomerada em três estágios de seleção (setor censitário, domicílio e o indivíduo). Para determinar o tamanho amostral partiu-se de uma prevalência de $14,5 \%$ de pobreza extrema ${ }^{9}$, de forma que uma amostra composta por no mínimo 1000 domicílios asseguraria a obtenção de estimativas para proporções sob níveis de precisão máximos iguais a $5 \%$. No primeiro estágio 75 setores foram determinados por meio de seleção sistemática com probabilidades proporcionais ao tamanho da Base Operacional Geográfica (BOG) de 2000 do IBGE, do total de 322 setores do referido distrito, garantindo a representatividade por estrato de renda, por faixa etária (crianças de seis a trinta meses, adolescentes de 12 a 18 anos e adultos) e sexo.

Antes da seleção dos domicílios foi realizada, entre outubro de 2004 e janeiro de 2005, uma varredura completa dos DPP dos 75 setores censitários selecionados, para localizar os endereços e identificar os DPP com crianças e adolescentes. Com esse levantamento foi possível estratificar os DPP de cada setor nos seguintes estratos: (a) DPP só com adultos, (b) DPP com adultos e adolescentes, (c) DPP com adultos e crianças e (d) DPP com adultos, adolescentes e crianças. Em cada estrato de cada setor, os DPP foram selecionados com eqüiprobabilidade e o tamanho da amostra de DPP, previamente fixado em 15 DPP por setor, foi alocado de forma a assegurar um mínimo de dois DPP por estrato e setor, além de um tamanho global da amostra de DPP que permitisse estimar os parâmetros desejados para crianças e adolescentes. No terceiro estágio, em cada DPP selecionado no segundo estágio foi selecionado um morador de cada grupo (criança, adolescente ou adulto) com eqüiprobabilidade dentre o total de moradores do grupo.

O tamanho final da amostra foi de 1.085 domicílios, com um total de $4 \%$ de perdas. Destes, 402 (37\%) domicílios apresentavam crianças na faixa etária de seis a trinta meses, o que representa uma perda de $7,6 \%$ em relação ao número de domicílios com crianças amostrados $(n=435)$. A coleta de dados foi realizada no período de maio a dezembro de 2005.

A equipe de entrevistadores foi composta 
por quinze moradores da comunidade, com no mínimo segundo grau completo, e por cinco nutricionistas (aferição das informações de crianças). Antes de iniciar a coleta de dados foram realizadas quatro etapas de treinamento para aplicação do questionário e das medidas antropométricas.

O estado nutricional das crianças foi avaliado através da aferição das medidas de peso e comprimento/estatura, seguindo os procedimentos recomendados pela literatura ${ }^{10}$. Para aferir o peso, foi utilizada uma balança digital do tipo "pesa-bebê", da marca "FILIZOLA", com variação de 1 grama, recomendada para as crianças menores de 2 anos de idade, sendo a criança pesada sentada ou deitada, sem roupa e acessórios (fralda, chupetas, chuquinhas, etc). $\mathrm{O}$ comprimento foi aferido com auxílio de um infantômetro de madeira portátil, estando à criança deitada em um colchão próprio para aferição, com a cabeça posicionada próxima à prancha imóvel, mantendo-a paralela à régua, com os joelhos pressionados, e os pés juntos com tornozelos em ângulo de $90^{\circ}$, mantidos pela prancha móvel, fazendo-se assim leitura. Para as crianças maiores de 24 meses, o peso foi coletado utilizando uma balança antropométrica digital, do tipo plataforma, da marca "PLENNA", com variação de 10 gramas, sendo a criança pesada em pé, sem roupas, sapatos e acessórios. A estatura foi aferida com o infantômetro na posição vertical, com a criança posicionada ereta, com os pés juntos e os braços estendidos ao longo do corpo, acertando a cabeça de forma que o plano de Frankfort ficasse paralelo ao chão. O comprimento/estatura foi aferido em duplicata.

As crianças foram avaliadas segundo os índices antropométricos peso-para-idade $(\mathrm{P} / \mathrm{I})$, comprimento/estatura-para-idade (E/I) e peso-para-comprimento/estatura (P/E), de acordo com o sexo e faixa etária, utilizando como referência a distribuição proposta pela Organização Mundial de Saúde em $2006^{11}$. Sendo assim, foram classificadas como eutróficas as crianças cujos índices $\mathrm{P} / \mathrm{I}, \mathrm{E} / \mathrm{I} \mathrm{e} \mathrm{P} / \mathrm{E}$ foram superiores a -2,0 e inferiores $a+2,0$ escores- $z$ e, em déficit, aquelas que apresentaram valores iguais ou inferiores a -2,0 escores- $z$ da mediana da curva de referência (variáveis dicotômicas) $^{12}$.

Para avaliar a influência dos indicadores socioeconômicos e do perfil de saúde no estado nutricional utilizou-se o modelo hierárquico dos determinantes da desnutrição em três níveis explicativos: $1^{\circ}$ - determinantes básicos, $2^{\circ}$ - intermediários e $3^{\circ}$ imediatos (Figura 1).

Para a avaliação dos determinantes básicos e intermediários foi aplicado um questionário com um adulto da família,
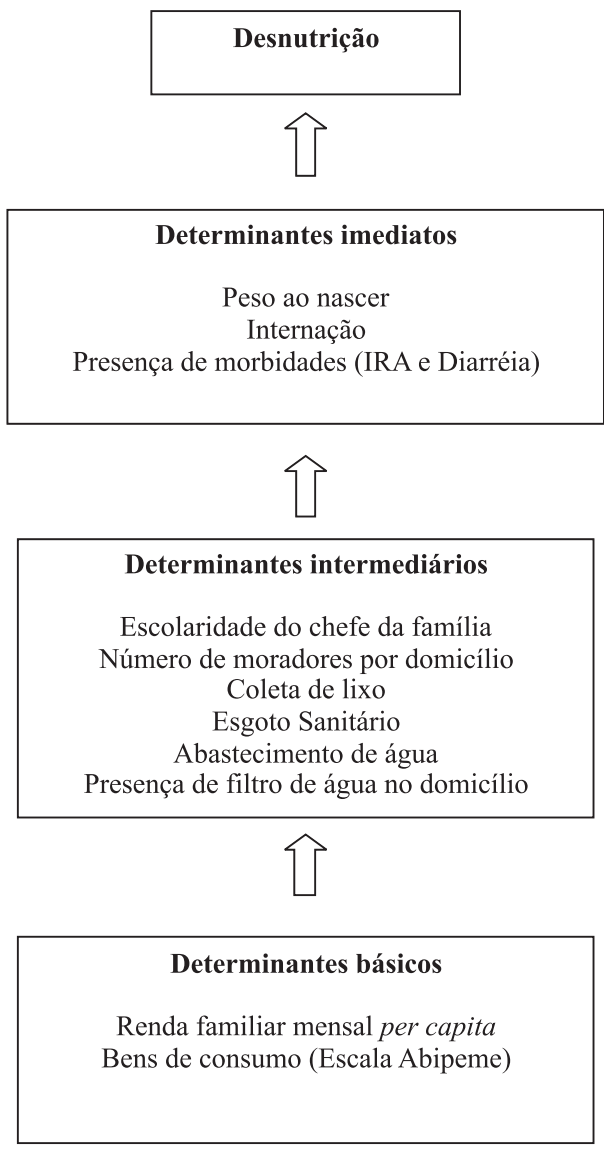

Figura 1 - Modelo hierarquizado dos determinantes da desnutrição entre crianças residentes em área de prevalência elevada de insegurança alimentar. Campos Elíseos/ Duque de Caxias, Rio de Janeiro, 2005.

Figure 1 - Hierarchical model of determinants of malnutrition among children living in area with high prevalence of food insecurity. Campos Elíseos/Duque de Caxias, Rio de Janeiro, 2005. 
avaliando as informações do domicílio e características familiares como as condições de moradia (abastecimento de água, coleta de lixo, esgoto sanitário, presença de filtro de água), renda familiar mensal per capita (total de rendimentos da família dividido pelo número de moradores que dependiam desta renda), número de moradores por domicílio, escolaridade do chefe da família ou cônjuge e a classificação socioeconômica da Associação Brasileira de Pesquisa de Mercados (avalia a escolaridade do chefe da família, o quantitativo de aparelhos domésticos, de automóvel, de banheiro e de empregada doméstica) (Abipeme) ${ }^{13}$.

Em relação aos determinantes imediatos foram considerados aqueles relacionados à criança: peso ao nascer (informação no cartão da criança), internação (criança que foi internada pelo menos uma noite nos últimos seis meses anteriores à entrevista) $\mathrm{e}$ morbidades como diarréia (últimos quinze dias anteriores à entrevista) e Infecção respiratória aguda (IRA) (existência de tosse, febre, chiado no peito e dificuldade para respirar nos últimos quinze dias).

Para a análise estatística foram estimados os valores médios e os respectivos valores de desvio padrão e distribuição percentual, de acordo com as categorias de cada variável para caracterizar a população estudada. Foi construído um modelo hierárquico por meio de regressão logística com as variáveis relacionadas aos determinantes básicos, intermediários e imediatos da desnutrição. Na primeira etapa, foram testados modelos univariados, estimando-se os valores das Razões de Chance brutas (Odds Ratio - OR), com intervalo de confiança de $95 \%$. Em seguida, realizou-se análise multivariada em cada nível hierárquico, ajustando os modelos pelas variáveis associadas significativamente ao nível de $5 \%$, com os desenlaces testados (índices antropométricos), e as variáveis que permaneceram associadas em cada nível constituíram o modelo hierárquico final (OR ajustado). As análises não foram ajustadas por sexo e idade por não apresentarem diferença significativa no modelo logístico univariado independente do índice antropométrico ( $\mathrm{p}>0,05)$.

Os parâmetros e os respectivos intervalos de confiança de $95 \%$ (IC 95\%) foram estimados para os dados expandidos, considerando-se o efeito do desenho amostral por conglomerado. O banco de dados foi elaborado e duplamente digitado por equipe previamente treinada e todos os procedimentos de análise realizados no programa Stata $9.0^{14}$.

Este estudo foi aprovado pelo Comitê de Ética em Pesquisa da Universidade do Estado do Rio de Janeiro.

\section{Resultados}

A amostra estudada foi constituída de 402 crianças, sendo 198 (49\%) do sexo feminino e 204 (51\%) do sexo masculino. Na Tabela 1 observa-se que as famílias são de baixa renda e $54,5 \%$ dos chefes das famílias apresentam escolaridade inferior a oito anos de estudo. Em relação às condições de moradia, a maioria dos domicílios tinha acesso à rede pública de abastecimento de água, de esgoto sanitário e coleta de lixo de forma regular. Apenas 57,3\% dos domicílios possuíam filtro para tratamento de água.

$\mathrm{Na}$ análise dos dados antropométricos $2,8 \%, 8,6 \%$ e $3,3 \%$ das crianças apresentaram déficit nutricional considerando-se os índices $\mathrm{P} / \mathrm{I}, \mathrm{E} / \mathrm{I}$ e $\mathrm{P} / \mathrm{E}$, respectivamente. A prevalência de baixo peso ao nascer foi de $11,4 \%$ e, em relação ao histórico de doenças, observou-se uma prevalência elevada de diarréia e IRA na população estudada. Encontrou-se também que 12,2\% das crianças foram internadas pelo menos por uma noite nos seis meses anteriores à entrevista (Tabela 1).

Os resultados da análise univariada revelaram que, entre os determinantes básicos, a renda familiar mensal per capita associou-se aos índices P/I, E/I e P/E. O número de moradores por domicílio e IRA associou-se ao déficit no índice P/I. Em relação ao índice E/I, os determinantes intermediários que apresentaram associação 
Tabela 1 - Tamanho da amostra (N), percentuais expandidos (\%) e respectivos intervalos de confiança das características socioeconômicas das famílias, dos índices antropométricos e do perfil de saúde de crianças de seis a trinta meses. Campos Elíseos/Duque de Caxias, Rio de Janeiro, 2005.

Table 1 - Sample size ( $N$ ), expanded percentage (\%) and respective confidence intervals of socioeconomic characteristics of families, of nutritional deficits and health of children with six to thirty months. Campos Elíseos/Duque de Caxias, Rio de Janeiro, 2005.

\begin{tabular}{|c|c|c|c|}
\hline Variáveis & $\mathrm{N}^{*}$ & $\%$ & $\begin{array}{c}\text { Intervalo de } \\
\text { confiança } 95 \%\end{array}$ \\
\hline Renda familiar mensal per capita ** & 402 & & \\
\hline $\begin{array}{l}0 \text { a } 50,00 \text { reais } \\
50,10 \text { a } 105,00 \text { reais } \\
105,10 \text { a } 200,00 \text { reais } \\
\text { Acima de } 200,00 \text { reais }\end{array}$ & & $\begin{array}{l}24,5 \\
23,5 \\
28,8 \\
23,2\end{array}$ & $\begin{array}{l}19,0-30,0 \\
17,9-28,9 \\
23,2-34,4 \\
17,1-29,3\end{array}$ \\
\hline Escolaridade do chefe da família & 387 & & \\
\hline $\begin{array}{l}\text { Analfabeto } \\
\text { Básico incompleto ( }<8 \text { anos) } \\
\text { Básico completo (8-10 anos) } \\
\text { Fundamental completo ( } 11-14 \text { anos) } \\
\text { Universitário ( } \geq 15 \text { anos) }\end{array}$ & & $\begin{array}{c}3,1 \\
51,4 \\
24,0 \\
20,9 \\
0,4\end{array}$ & $\begin{array}{c}1,4-4,9 \\
44,7-58,1 \\
18,7-29,3 \\
14,8-27,1 \\
0,1-10,0\end{array}$ \\
\hline Número de pessoas por domicílio & 402 & & \\
\hline $\begin{array}{l}1-4 \\
5-8 \\
\geq 8\end{array}$ & & $\begin{array}{c}51,3 \\
40,9 \\
7,8\end{array}$ & $\begin{array}{c}44,8-57,8 \\
34,6-47,1 \\
3,9-11,5\end{array}$ \\
\hline Escala Abipeme ${ }^{* * *}$ & 402 & & \\
\hline $\begin{array}{l}\text { A } \\
B \\
C \\
D \\
\text { E }\end{array}$ & & $\begin{array}{c}1,3 \\
4,2 \\
39,3 \\
41,8 \\
13,2\end{array}$ & $\begin{array}{c}0,2-2,8 \\
1,9-6,5 \\
31,9-46,6 \\
33,9-49,7 \\
8,2-18,2\end{array}$ \\
\hline Saneamento Básico & 402 & & \\
\hline Coleta de lixo $0^{* * * *}$ & & 85,9 & $81,8-90,1$ \\
\hline Abastecimento de água**** & & 67,7 & $61,9-73,5$ \\
\hline Tratamento de esgoto**** & & 65,9 & $56,2-75,6$ \\
\hline Presença de filtro para tratamento de água & & 57,3 & $50,8-63,7$ \\
\hline Peso/ldade & 402 & & \\
\hline Déficit nutricional ( $\leq-2,0$ escores- $z$ ) & & 2,8 & $0,7-4,8$ \\
\hline Estatura/ldade & 392 & & \\
\hline Déficit nutricional ( $\leq-2,0$ escores- $z$ ) & & 8,6 & $5,3-11,8$ \\
\hline Peso/Estatura & 391 & & \\
\hline Déficit nutricional ( $\leq-2,0$ escores-z) & & 3,3 & $0,5-6,2$ \\
\hline Crianças com baixo peso ao nascer & 382 & 11,4 & $7,7-15,0$ \\
\hline Episódio de diarréia ${ }^{* * * * *}$ & 397 & 23,6 & $18,3-28,9$ \\
\hline Episódios de IRA $* * * * * *$ & 399 & 14,3 & $9,8-18,8$ \\
\hline Ocorrência de Internação******* & 398 & 12,2 & $8,1-16,3$ \\
\hline
\end{tabular}

*Os valores diferem devido a perdas em cada variável; Different totals due to missing values in each variable;

** distribuição em quartis. Quartile distribuition;

***Associação Brasileira de Pesquisa de Mercados; Brazilian Association of Market Survey;

**** executado pelo serviço público. Executed by public service system;

***** episódio de diarréia nos últimos quinze dias; Diarrhea in past fifteen days;

****** Infecção respiratória aguda - presença de febre, tosse, chiado no peito e dificuldade para respirar nos últimos 15 dias; Acute respiratory infection - defined by fever, cough, wheezing, and difficulty to breath in past fifteen days;

******* internação nos últimos seis meses; hospitalization in past six months. 
Tabela 2 - Razões de Chance (odds ratio-OR) bruta e respectivos intervalos de confiança (IC 95\%) para os determinantes dos déficits dos índices antropométricos de crianças de seis a trinta meses. Campos Elíseos/ Duque de Caxias, Rio de Janeiro, 2005.

Table 2 - Crude odds ratio (OR) and respective confidence intervals (95\% IC) for determinants of anthropometric index deficiencies for children with six to thirty months. Campos Elíseos/ Duque de Caxias, Rio de Janeiro, 2005.

\begin{tabular}{|c|c|c|c|}
\hline Variáveis & $\begin{array}{c}\text { Peso/Idade } \\
\text { OR bruto (IC 95\%) }\end{array}$ & $\begin{array}{c}\text { Estatua/ldade } \\
\text { OR bruto (IC 95\%) }\end{array}$ & $\begin{array}{c}\text { Peso/Estatura } \\
\text { OR bruto (IC 95\%) }\end{array}$ \\
\hline \multicolumn{4}{|l|}{ Determinantes básicos } \\
\hline Renda familiar mensal per capita* & $2,35(1,03-5,36)$ & $1,73(1,12-2,68)$ & $3,28(1,49-7,22)$ \\
\hline Escala Abipeme** & $1,3(0,68-1,55)$ & $1,06(0,83-1,36)$ & $0,92(0,51-1,66)$ \\
\hline \multicolumn{4}{|l|}{ Determinantes intermediários } \\
\hline \multicolumn{4}{|l|}{ Escolaridade do chefe da família } \\
\hline Maior que sete anos & 1,0 & 1,0 & 1,0 \\
\hline Menor que sete anos & $1,49(0,32-6,88)$ & $0,31(0,11-0,87)$ & $1,57(0,59-4,23)$ \\
\hline \multicolumn{4}{|l|}{ Número de moradores por domicílio } \\
\hline $1-4$ & 1,0 & 1,0 & 1,0 \\
\hline$>4$ & $2,10(1,01-4,35)$ & $1,24(0,67-2,28)$ & $2,09(0,98-4,45)$ \\
\hline \multicolumn{4}{|l|}{ Coleta de lixo } \\
\hline Com coleta & 1,0 & 1,0 & 1,0 \\
\hline Sem coleta & $1,65(0,30-9,01)$ & $1,58(0,61-4,08)$ & $0,73(0,14-3,67)$ \\
\hline \multicolumn{4}{|l|}{ Esgoto sanitário } \\
\hline Com rede pública & 1,0 & 1,0 & 1,0 \\
\hline Sem rede pública & $0,83(0,19-3,49)$ & $0,73(0,29-1,80)$ & $1,12(0,39-3,24)$ \\
\hline \multicolumn{4}{|l|}{ Abastecimento de água } \\
\hline Com rede pública & 1,0 & 1,0 & 1,0 \\
\hline Sem rede pública & $1,84(0,36-9,30)$ & $0,89(0,36-2,21)$ & $2,12(0,78-5,78)$ \\
\hline \multicolumn{4}{|c|}{ Presença de filtro para tratamento de água } \\
\hline Com filtro & 1,0 & 1,0 & 1,0 \\
\hline Sem filtro & $1,68(0,33-8,51)$ & $3,17(1,28-7,83)$ & $0,79(0,29-2,17)$ \\
\hline \multicolumn{4}{|l|}{ Determinantes imediatos } \\
\hline \multicolumn{4}{|l|}{ Internação hospitalar } \\
\hline Não & 1,0 & 1,0 & 1,0 \\
\hline Sim & $0,68(0,12-3,93)$ & $0,37(0,14-0,98)$ & $0,68(0,19-2,42)$ \\
\hline \multicolumn{4}{|l|}{ Peso ao nascer } \\
\hline$\geq 2.500 \mathrm{~g}$ & 1,0 & 1,0 & 1,0 \\
\hline$<2.500 \mathrm{~g}$ & $3,75(0,74-19,0)$ & $3,64(1,41-9,38)$ & $4,63(1,56-13,68)$ \\
\hline \multicolumn{4}{|l|}{ Ocorrência de Diarréia ${ }^{* * *}$} \\
\hline Ausência & 1,0 & 1,0 & 1,0 \\
\hline Presença & $0,58(0,11-2,85)$ & $0,50(0,20-1,21)$ & $0,60(0,21-1,72)$ \\
\hline \multicolumn{4}{|l|}{ Ocorrência de IRA**** } \\
\hline Ausência & 1,0 & 1,0 & 1,0 \\
\hline Presença & $0,09(0,01-0,80)$ & $1,18(0,34-4,04)$ & $1,66(0,47-5,85)$ \\
\hline \multicolumn{4}{|l|}{ Determinantes imediatos } \\
\hline Idade & $1,06(0,97-1,15)$ & $1,04(0,98-1,10)$ & $0,95(0,88-1,03)$ \\
\hline \multicolumn{4}{|l|}{ Sexo } \\
\hline Masculino & 1,0 & 1,0 & 1,0 \\
\hline Feminino & $3,27(0,74-14,4)$ & $1,23(0,53-2,86)$ & $0,92(0,34-2,48)$ \\
\hline
\end{tabular}

*considerando todos os rendimentos da família no mês anterior a entrevista, avaliada como variável contínua; Income of family in month previous to interview, evaluated as a continuous variable;

** Associação Brasileira de Pesquisa de Mercados analisada como variável ordinal (categorias A (referência), B, C, D e E); Brazilian Association of Market Survey evaluated as an ordinal variable ( $A$ (reference category), B, C, D and E);

*** episódio de diarréia nos últimos quinze dias; Diarrhea in past fifteen days;

**** infecção respiratória aguda - presença de febre, tosse, chiado no peito e dificuldade para respirar nos últimos 15 dias. Acute breath infection - defined by fever, cough, wheezing, and difficulty to breath in past fifteen days. 
Tabela 3 - Razões de Chance (odds ratio-OR) ajustadas e respectivos intervalos de confiança (IC 95\%) para os determinantes dos déficits dos índices antropométricos de crianças de seis a trinta meses. Campos Elíseos/ Duque de Caxias, Rio de Janeiro, 2005.

Table 3 - Adjusted odds ratio (OR) and respective confidence intervals (95\% IC) for determinants of anthropometric index deficiencies for children with six to thirty months. Campos Eliseos/Duque de Caxias, Rio de Janeiro, 2005.

\begin{tabular}{|c|c|c|}
\hline \multirow[t]{2}{*}{ Variáveis } & \multicolumn{2}{|c|}{ Índice antropométrico Peso/Idade } \\
\hline & OR ajustado & IC $95 \%$ \\
\hline \multicolumn{3}{|l|}{ Ocorrência de IRA* } \\
\hline Ausência & 1,0 & \\
\hline \multirow[t]{3}{*}{ Presença } & 0,08 & $0,01-0,78$ \\
\hline & \multicolumn{2}{|c|}{ Índice antropométrico Estatura/ldade } \\
\hline & OR ajustado & IC $95 \%$ \\
\hline \multicolumn{3}{|l|}{ Peso ao nascer } \\
\hline$\geq 2.500 \mathrm{~g}$ & 1,0 & \\
\hline \multirow[t]{3}{*}{$<2.500 \mathrm{~g}$} & 3,55 & $1,36-9,24$ \\
\hline & \multicolumn{2}{|c|}{ Îndice antropométrico Peso/Estatura } \\
\hline & OR ajustado & IC $95 \%$ \\
\hline Renda familiar mensal per capita** & 3,55 & $1,51-8,23$ \\
\hline \multicolumn{3}{|l|}{ Peso ao nascer } \\
\hline$\geq 2.500 \mathrm{~g}$ & 1,0 & \\
\hline$<2.500 \mathrm{~g}$ & 8,31 & $1,39-49,34$ \\
\hline
\end{tabular}

foram a escolaridade do chefe da família e a presença de filtro de água no domicílio; os determinantes imediatos foram o peso ao nascer e internação. Observou-se também que as crianças que nasceram com baixo peso apresentavam maior chance de déficit no índice P/E (Tabela 2).

Na Tabela 3 encontram-se os determinantes do déficit nos índices antropométricos após o ajuste do modelo. Identificou-se que a IRA associou-se significativamente ao déficit no índice $\mathrm{P} / \mathrm{I}$. A renda familiar mensal per capita permaneceu no modelo final, sendo estatisticamente associada ao déficit no índice $\mathrm{P} / \mathrm{E}$. O baixo peso ao nascer, quando comparado com o peso adequado, elevou cerca de três vezes $(\mathrm{OR}=3,55$; IC 95\%: $1,36-9,24)$ e de oito vezes $(\mathrm{OR}=8,31$; IC 95\%: 1,39-49,34) a chance de a criança apresentar déficit nos índice E/I e P/E, respectivamente.

\section{Discussão}

Apesar de o município de Duque de Caxias concentrar um grande número de indústrias e comércio e possuir o sexto maior Produto Interno Bruto (PIB) (1999 - 2003) no ranking nacional e o segundo maior do Estado do Rio de Janeiro ${ }^{15}$, este estudo revela que as famílias residentes no maior distrito do município se encontram em condições socioeconômicas inadequadas. Isto se reflete no nível de escolaridade do chefe e no baixo poder aquisitivo da família. Apesar do quadro aparentemente favorável das variáveis relacionadas ao saneamento básico, os dados encontrados revelam alto percentual de famílias com condições insuficientes de abastecimento de água, de esgoto sanitário e de tratamento da água para o consumo individual.

Dos déficits antropométricos avaliados destacou-se a prevalência do déficit de esta- 
tura (E/I) de 8,6\% maior do que o encontrado no estudo de Monteiro \& Conde $^{16}$ (2000), analisando desnutrição na cidade de São Paulo nos anos de 1995/1996 (2,4\%), como também no estudo de Zöllner \& Fisberg ${ }^{17}$ (2006) realizado em creches da prefeitura de São Paulo (5,2\%), e no estudo realizado na macro-região Nordeste e em municípios do semi-árido do Brasil $(6,6 \%)^{18}$. Apesar de o déficit de $\mathrm{P} / \mathrm{E}$ possuir pouca expressão em termos epidemiológicos em crianças brasileiras ${ }^{19}$, nesta amostra observou-se uma prevalência de $3,3 \%$, sugerindo que essas crianças apresentam um comprometimento dos tecidos muscular e/ou gorduroso. Por outro lado, os resultados revelam que o déficit nutricional no índice P/I de 2,8\% encontrado neste estudo foi inferior ao observado por Gigante et al ${ }^{20}$ (2003), no estudo realizado com as crianças nascidas em Pelotas na década de 1990 (3,8\%), por Lira et al ${ }^{21}$ (2003) em crianças de áreas urbanas da Zona da Mata Meridional de Pernambuco $(6,8 \%)$ e por Monteiro et al ${ }^{18}$ (2006) na região Nordeste e em municípios do semi-árido do Brasil (5,6\%).

Analisando o resultado final do modelo hierárquico proposto para a população de Campos Elíseos (Tabela 3) observou-se que a renda familiar mensal per capita foi o único indicador socioeconômico associado significativamente ao déficit nutricional no índice $\mathrm{P} / \mathrm{E}$, que expressa um processo agudo de má nutrição, refletindo predominantemente as condições ambientais sob as quais a criança vive no momento ${ }^{22}$. Os determinantes básicos relacionados no presente trabalho não se associaram aos outros índices antropométricos P/I e E/I. Uma possível explicação para este resultado seria a relativa homogeneidade da amostra quanto às condições desfavoráveis dos indicadores socioeconômicos da população em questão, repercutindo na prevalência elevada de insegurança alimentar, como descrito por Pimentel ${ }^{7}$ (2007).

A forte associação encontrada entre $\mathrm{O}$ baixo peso ao nascer e o déficit no índice $\mathrm{P} / \mathrm{E}$ tem sido discutido por outros autores ${ }^{23-25}$. $\mathrm{O}$ baixo peso ao nascer esteve associado ainda ao déficit no índice E/I, semelhante ao observado por Oliveira et $\mathrm{al}^{27}$ (2006) no estudo com crianças do Estado da Bahia $(\mathrm{OR}=3,6$; IC 95\%: 1,72-7,70). De fato, este indicador contribui para o déficit de crescimento pós-natal, além de comprometer a amamentação e aumentar a vulnerabilidade para a ocorrência de doenças na infãncia ${ }^{28}$. A OMS considera o baixo peso ao nascer o mais importante determinante isolado das chances de sobrevivência da criança nos primeiros meses de vida ${ }^{29}$.

Cabe destacar que o desenho de estudo transversal traz limitações importantes, sendo uma delas a impossibilidade de apresentar uma relação de causa e efeito entre as variáveis estudadas. Desta forma os resultados observados não devem ser entendidos como fatores de risco para a desnutrição e sim como associações entre os eventos. Outra limitação deste estudo se deve ao fato de a amostra estudada ter sido calculada para obtenção de estimativas de proporções, de modo que é possível que a mesma não tenha sido suficiente para encontrar algumas associações com outros fatores, além da renda familiar e do peso ao nascer.

Este é o primeiro estudo de base populacional avaliando informações da saúde infantil em Campos Elíseos, no município de Duque de Caxias, e enfatiza a situação de pobreza desta região, apesar da concentração de indústrias de grande porte que colaboram para grande parte do PIB do município. Atuar nos determinantes sociais é, indubitavelmente, fundamental para diminuir os índices de insegurança alimentar e de desnutrição infantil. Entretanto, este estudo revelou que ações de intervenção nos determinantes imediatos, priorizando o nível básico de atenção da saúde, podem minimizar o déficit nutricional infantil de populações caracterizadas por desigualdades sociais marcantes.

Por fim, adequar e aprimorar as atividades realizadas, principalmente pela rede pública dos postos de saúde, como os programas de vigilância nutricional da gestante e da criança, e planejamento familiar, entre tantos outros que reduzem a ocorrência do baixo peso ao nascer, podem contribuir para minimizar a desnutrição infantil. 


\section{Referências}

1. Fundo das Nações Unidas para a Infância. Desnutrição: causas, conseqüências e soluções. Brasília (DF); 1998.

2. Martins MFD, Costa JSD, Saforcada ET, Cunha MDC. Qualidade do ambiente e fatores associados: um estudo em crianças de Pelotas, Rio Grande do Sul, Brasil. Cad Saúde Pública. 2004; 20 (3): 710-8.

3. Monteiro CA, Freitas ICM. Evolução de condicionantes socioeconômicas da saúde na infância na cidade de São Paulo (1984-1996). Rev Saúde Pública. 2000; 34 (6S): S8-12.

4. Ribas DLB, Philippi ST, Tanaka AC, Zorzatto JR. Saúde e estado nutricional infantil de uma população da região Centro-Oeste do Brasil. Rev Saúde Pública. 1999; 33 (4): 358-65.

5. Onis M, Blösser M. The World Health Organization Global Database on Child Growth and Malnutrition: methodology and applications. Intern Journal of Epidemiology 2000; 32: 518-26.

6. Conselho Nacional de Segurança Alimentar. Princípios e diretrizes de uma política de segurança alimentar. Brasília: Editora Positiva; 2004.

7. Pimentel PG. Condições socioeconômicas, insegurança alimentar no domicílio associada com indicadores antropométricos de crianças menores de 30 meses residentes na região metropolitana do Rio de Janeiro. [dissertação de mestrado]. Rio de Janeiro: Universidade Federal Do Rio De Janeiro, Centro de Ciências da Saúde, Instituto De Nutrição; 2007.

8. Segall-Corrêa AM, Pérez-Escamilla R., Maranha LK, Sampaio MFA, Yuyama L, Alencar F, et al. Projeto: Acompanhamento e avaliação da segurança alimentar de famílias brasileiras: validação de metodologia e de instrumento de coleta de informação. Campinas: Departamento de Medicina Preventiva e Social, Universidade Estadual de Campinas/ Organização Pan-Americana da Saúde/Ministério de Saúde; 2003. (Relatório Técnico).

9. Monteiro CA. A dimensão da pobreza, da desnutrição e da fome no Brasil: implicações para políticas públicas. Instituto Nacional de Altos Estudos. Seminário Especial Fome e Pobreza, 2003. Disponível em URL: http:/ / forumnacional.org.br (Acessado em 20 de novembro de 2006).

10. Lohman TG, Roche AF, Martorell R, editors. Anthropometric standardization reference manual. Champaing (IL): Human Kinectics Books; 1988.

11. World Health Organization. Child Growth Standards. Methods and Development, World Health Organization; 2006. Disponível em URL: http:/ / www.who.int/ childgrowth/en/ (Acessado em agosto de 2006).

12. World Health Organization. The Use and interpretation of Anthropometry Physical status: the use and interpretation of anthropometry: report of a WHO expert committee. Geneva, World Health Organization; 1995 (WHO Technical Report Series, No. 854).
13. Associação Brasileira de Institutos de Pesquisa de Mercado (Abipeme). Classificação socioeconômica critério Abipeme. http://www.ufrn.br/sites/fonaprace/ perfilanexo3.doc (Acessado em 10 de janeiro de 2007).

14. Stata Corporation: version 9.0. Texas: College Station; 2006.

15. Instituto Brasileiro de Geografia e Estatística 1999-2003. Produto Interno Bruto dos Municípios; 2005. Disponível em URL: http://www.ibge.gov.br/ (Acessado em 20 de janeiro de 2007).

16. Monteiro CA, Conde WL. Tendência secular da desnutrição e da obesidade na infância na cidade de São Paulo (1974-1996). Rev Saúde Pública 2000; 36(6): 52-61.

17. Zöllner CC, Fisberg RM. Estado nutricional e sua relação com fatores biológicos, sociais e demográficos de crianças assistidas em creches da Prefeitura do Município de São Paulo. Rev Bras Saúde Matern Infant 2006; 6(3): 319-28.

18. Monteiro CA, Conde WL, Konno SC. Análise do inquérito "Chamada Nutricional 2005" realizado pelo Ministério do Desenvolvimento Social e Combate à Fome e Ministério da Saúde. Núcleo de Pesquisas Epidemiológicas em Nutrição e Saúde e Departamento de Nutrição da Faculdade de Saúde Pública da Universidade de São Paulo; 2006.

19. Sociedade Civil Bem Estar Familiar no Brasil, BENFAM. Pesquisa Nacional Sobre Demografia e Saúde 1996, 1997. Cap 9 (Amamentação e situação nutricional de mães e crianças): 125-38.

20. Gigante DP, Victora CG, Araújo CLP, Barros FC. Tendências no perfil nutricional das crianças nascidas em 1993 em Pelotas, Rio Grande do Sul, Brasil: análises longitudinais. Cad Saúde Pública. 2003; 19 (S1): S141-7.

21. Lira PIC, Lima MC, Silva GAP, Romani SAM, Eickmann $\mathrm{SH}$, Alessio MLM, et al. Saúde e nutrição de crianças de áreas urbanas da Zona da Mata Meridional de Pernambuco: resultados preliminares de um estudo de coorte. Rev Bras Saude Mater Infant 2003; 3 (4): 463:72.

22. Olinto MT, Victora CG, Barros FC, Tomasi E. Determinantes da desnutrição infantil em uma população de baixa renda: um modelo de análise hierarquizado. Cad Saúde Pública. 1993; 9 (S1): S14-27.

23. Ong KK, Ahmed ML, Emmett PM, Preece MA, Dunger DB. Association between postnatal catch-up growth and obesity in childhood: prospective cohort study. BMJ. 2000; 320 (7240): 967-71.

24. Ong KK, Dunger DB. Birth weight, infant growth and insulin resistence. Eur. J. Endocrinol. 2004; 151(S3): S1319 .

25. Ravelli GP, Stein ZA \& Susser MW. Obesity in young men after famine exposure in utero and early infance. New England Journal of Medicine. 1976; 295: 349-53. 
26. Martins IS, Marinho SP, Oliveira DC, Araújo EAC. Pobreza, desnutrição e obesidade: inter-relação de estados nutricionais de indivíduos de uma mesma família. Ciência \& Saúde Coletiva 2007: 12(6): 1553-65.

27. Oliveira VA, Assis AMO, Pinheiro SMC, Barreto ML. Determinantes dos déficits ponderal e de crescimento linear de crianças menores de dois anos. Rev Saúde Pública. 2006; 40 (5): 874-82.
28. Romani SAM, Lira PIC. Fatores determinantes do crescimento infantil. Rev Bras Saúde Matern Infant 2004, 4(1): 15-23.

29. Ministério da Saúde. Guia alimentar para crianças menores de 2 anos; 2002.

Recebido em: 26/11/07

Versão final reapresentada em: 16/05/08

Aprovado em: 30/05/08 